\title{
Justicia organizacional, engagement en el trabajo y comportamientos de ciudadanía organizacional: una combinación ganadora*
}

\author{
Organizational Justice, Work Engagement and Organizational \\ Citizenship Behaviors: A Winning Combination
}

Recibido: febrero 11 de 2013 | Revisado: marzo 14 de 2014 | Aceptado: marzo 14 de 2014

\author{
RAMÓN LUIS RODRÍGUEZ MONTALBÁN ** \\ Miguel MARTÍNEZ LUGO *** \\ Universidad Carlos Albizu, Puerto Rico \\ MARISA SALANOVA SORIA ***** \\ Universitat Jaume I, Castellón, España
}

doi:10.11144/Javeriana.UPSY13-3.joet

Para citar este artículo: Rodríguez, R. L., Martínez, M., \& Salanova, M.. (2014). Justicia organizacional, engagement en el trabajo y comportamientos de ciudadanía organizacional: una combinación ganadora. Universitas Psychologica, 13(3), 961-974. http://dx.doi.org/10.11144/Javeriana.UPSY13-3.joet

* Artículo original resultado de investigación.

** Personal Docente Investigador. WoNT Research Team. Departamento de Psicología Social. Correo electrónico: ramon.rodriguez@uji.es

***** Departamento de Psicología Social. Directora WoNT Research Team. Correo electrónico: marisa. salanova@uji.es

****** Director del Programa de Psicología Industrial/ Organizacional. Recinto de San Juan de Puerto Rico. Correo electrónico: mmartinez@albizu.edu
RESUMEN

Se han realizado muchos estudios sobre la relación de la justicia organizacional y los comportamientos de ciudadanía organizacional. Sin embargo, todavía existen preguntas sobre los procesos psicológicos que explican esta relación. Este estudio pone a prueba el rol del mediador del engagement en el trabajo, como proceso psicológico que explica la relación entre la justicia organizacional y los comportamientos de ciudadanía organizacional. Una muestra de 144 empleados de múltiples sectores ocupacionales (servicios, ventas, entre otros) de Puerto Rico participó en el estudio. Los resultados de los modelos de ecuaciones estructurales con Partial Least Squares apoyan nuestra hipótesis: el engagement media en su totalidad la relación entre justicia y comportamientos de ciudadanía organizacional. Cuando las personas perciben que son tratadas en el trabajo de forma justa, se fomenta el engagement en el trabajo, lo que a su vez, predice de los comportamientos de ciudadanía organizacional.

Palabras clave

Justicia organizacional; engagement en el trabajo; comportamientos de ciudadanía organizacional

\section{A B S T R ACT}

Many studies have been conducted about the relationship of organizational justice and organizational citizenship behaviors. However, there still are questions regarding the psychological processes that explain the relationship. This study tests the mediating role of work engagement as a psychological process that explains the relationship between organizational justice and organizational citizenship behaviors. A sample of 144 employees from multiple occupational industries (service, sales, among others) in Puerto Rico participated in this study. The results from structural equation with Partial Least Squares support our hypothesis: work engagement completely mediates the relationship between organizational justice and organizational citizenship behaviors. When employees are treated in a fair way, work engagement is fostered, which in turn, predicts organizational citizenship behaviors. Keywords

Organizational justice; work engagement; organizational citizenship behaviors 
Hoy día muchas organizaciones están tratando de subsistir y poder hacer frente a la crisis económica mundial. Esta crisis ha traído consigo muchos cambios en los lugares de trabajo. Para poder ajustarse a estos cambios, las organizaciones requerirán de una fuerza trabajadora involucrada con su trabajo y que ponga de manifiesto los comportamientos necesarios para que las organizaciones puedan superar estos eventos de crisis. ¿Qué pueden hacer las organizaciones para generar estas conductas en los empleados²? El propósito de este estudio es estudiar las relaciones que existen entre las percepciones individuales de la justicia organizacional, con el engagement en el trabajo y los comportamientos de ciudadanía organizacional.

\section{¿Qué es la justicia organizacional?}

Desde los tiempos de Platón y Aristóteles los seres humanos han estado preocupados por tratar de encontrar la justicia en diferentes facetas de la vida (Ryan, 1993). Existe un cúmulo de teorías sociales que pretenden brindar una explicación ontológica del concepto de la justicia, por ejemplo la teoría de la equidad (Adams, 1966), equidad en el intercambio social (Homans, 1961), expectativas en el intercambio social (Blau, 1964), normas de distribución múltiple (Leventhal, 1976); equidad en los procedimientos de disputas (Thibaut \& Walker, 1975) y la teoría del trato interpersonal (Bies \& Moag, 1986). Estas teorías han permitido entender la justicia como una construcción social (Colquitt, Conlon, Weson, Porter \& Ng, 2001). Cónsono con esta concepción, Greenberg (1987) acuña el concepto de la justicia organizacional como 'la percepción de los empleados respecto a lo que es justo en la organización'.

No es sino hasta que el trabajo seminal de Colquitt (2001) donde se integran las múltiples teorías de la justicia, elaborando un modelo de justicia organizacional compuesto por cuatro dimensiones: justicia distributiva, justicia procedimental, justicia

1 Para facilitar la lectura de este artículo, cuando se menciona las palabras empleado(s) y supervisor(es), participantes, autores e investigadores, se hace referencia tanto a hombres y mujeres. interpersonal y justicia informacional. La justicia distributiva, se refiere al contenido de las distribuciones y a la justicia de los fines o resultados alcanzados. La justicia procedimental, se refiere a la justicia de los medios utilizados para determinar las distribuciones. La justicia interpersonal se refiere a la sensibilidad social, que concierne al grado con que los supervisores tratan de forma respetuosa y digna a las personas afectadas por sus decisiones y procedimientos distributivos. Mientras que la justicia informacional se refiere a la explicación acerca del racional de las decisiones tomadas por los supervisores.

Utilizando el modelo de Colquitt (2001) se han realizado múltiples estudios con hallazgos de relevancia para las organizaciones, centrándose en las actitudes y conductas que emergen a partir de las percepciones de justicia organizacional. Por ejemplo, tal como se evidencia en varios estudios (Judge \& Colquitt, 2004; Moorman, 1991; Omar, 2006) las actitudes y los comportamientos de las personas en su lugar de trabajo, están influenciados por los juicios valorativos que hacen de las interacciones y de su entorno social. Por tanto, si estas valoraciones son positivas, es más probable que las personas se sientan más a gusto con el trabajo que hacen y donde lo hacen (Colquitt et al., 2001).

Cabe señalar que la justicia organizacional está relacionada de forma positiva y negativa con distintas variables que inciden en la conducta y actitudes de las personas. Por ejemplo, existe evidencia que la justicia organizacional está relacionada de forma negativa con las intenciones de abandono (Loi, Yang \& Diefendorff, 2009), burnout (Van Dierendonck, Schaufeli \& Buunk, 2001) y la tensión psicológica (Francis \& Barling, 2005). Del mismo modo, existe evidencia que demuestra que la justicia organizacional se relaciona positivamente con la satisfacción en el trabajo (Moorman, 1991), la confianza organizacional y el apoyo (DeConinck, 2010) y el engagement en el trabajo (Moliner, MartínezTur, Ramos, Peiró \& Cropanzano, 2008).

Es importante destacar que existe evidencia robusta, donde se pone de manifiesto la relación entre la justicia organizacional y los comportamientos de ciudadanía organizacional (CCO) (Farh, Podsakoff 
\& Organ, 1990; Konovsky \& Organ, 1996; Moorman, 1991; Moorman, Niehoff \& Organ, 1993; Niehoff \& Moorman, 1993). Sin embargo, aún hay preguntas relacionadas a los mecanismos psicológicos que expliquen la relación entre la justicia organizacional y los CCO. Tal como señalan Moorman y Byrne (2005) existen distintos mecanismos que pueden explicar esta relación, por lo que existen diferencias entre los investigadores sobre cuáles pueden ser los procesos psicológicos que ocurren para que se pongan de manifiesto los CCO. En esta investigación se propone el engagement en el trabajo como el posible mecanismo motivacional que explique la relación entre la justicia organizacional y los CCO.

\section{¿Qué es el engagement en el trabajo?}

El engagement en el trabajo se define como un estado mental positivo, relativamente persistente, relacionado con el trabajo y que se caracteriza por el vigor, la dedicación y la absorción (Schaufeli, Salanova, González-Romá \& Bakker, 2002). El vigor (dimensión conductual) conlleva altos niveles de energía y activación mental en el trabajo y el deseo de invertir esfuerzo en el trabajo que se está realizando, incluso cuando aparecen dificultades en el camino. La dedicación (dimensión afectiva) se refiere a una alta implicación laboral, junto con la manifestación de un sentimiento de entusiasmo, orgullo y reto por el trabajo. La absorción (dimensión cognitiva) implica estar totalmente concentrado en el trabajo, experimentar que el tiempo "pasa volando" y que se tienen dificultades en desconectarse o desligarse de lo que se está haciendo debido a esa alta concentración y disfrute.

Existe evidencia empírica de la relación entre el engagement en el trabajo y otras variables teóricas (actitudes y conductas). Los empleados engaged se sienten energizados, se perciben como personas autoeficaces (Salanova, Llorens \& Schaufeli, 2011); son capaces de crear su propia retroinformación positiva, apreciación, reconocimiento y éxito (Bakker, 2008); están más comprometidos con la organización (Hakanen, Bakker \& Schaufeli, 2006) y presentan conductas extrarrol (Salanova,
Agut \& Peiro, 2005; Salanova, Lorente, Chambel \& Martínez, 2011).

Cabe destacar que la evidencia empírica sobre la relación entre el engagement en el trabajo y las percepciones de justicia organizacional es muy escasa. En la literatura revisada, se destaca el estudio de Moliner et al. (2008) el cual evidencia la relación positiva y significativa entre la justicia organizacional y el engagement en el trabajo. Más recientemente Karatepe (2011) encontró una relación positiva entre la justicia procedimental y el engagement. Se entiende que la justicia organizacional puede fomentar el engagement en los empleados, dado que las percepciones de justicia pueden ser consideradas como un agente motivador de forma instrumental (para conseguir determinados recursos que motiven a los empleados) o a nivel relacional (para fomentar las relaciones saludables entre las personas dentro de la organización).

\section{¿Qué son los comportamientos de ciudadanía organizacional?}

Para la consecución de sus metas, las organizaciones necesitan que sus empleados, en determinadas ocasiones, vayan más allá de lo que se le es requerido en su puesto de trabajo y se comprometan personalmente en el logro de los objetivos colectivos, para que estos se tornen alcanzables. En complementariedad al buen desempeño de la tarea establecida, hoy las organizaciones están interesadas en un conjunto de comportamientos que se han designado con diversas etiquetas, siendo uno de estos los comportamientos de ciudadanía organizacional (Organ, Podsakoff \& Mackenzie, 2005).

El comportamiento de ciudadanía organizacional (CCO) es "aquel comportamiento individual que es discrecional, no reconocido directa o explícitamente por el sistema formal de recompensas y que promueve el funcionamiento efectivo de la organización" (Organ, 1988, p. 4). Cabe destacar que este comportamiento es de naturaleza discrecional, y no se considera un requerimiento específico de la descripción del puesto de trabajo. Por el contrario, este comportamiento involucra cierto grado de opción personal, de modo que la persona no resulte 
castigada si decide no comportarse de dicha forma. Es importante señalar que la función principal de los CCO es apoyar el ambiente social y psicológico en el cual se lleva a cabo el desempeño de la tarea.

Este constructo ha sido conceptuado por Organ (1997) en cinco dimensiones: Altruismo, Concienciación, Deportividad, Cortesía y Virtud Cívica. El altruismo se conforma por aquellos comportamientos espontáneos dirigidos a ayudar a otras personas con sus tareas o con algún problema relacionado con la organización. La concienciación se refiere a la asistencia al trabajo y al cumplimiento de las reglas y los procedimientos de la organización. La deportividad es la disposición de los empleados de tolerar condiciones de trabajo indeseables sin quejarse de estas. La cortesía se define como el comportamiento de consulta con otras personas antes de tomar decisiones que puedan afectar su trabajo. Por último, la virtud cívica incluye todas aquellas acciones que indican que el individuo participa, se involucra y se preocupa por la vida de la organización.

Tal como se ha destacado, el estudio de la justicia organizacional y los comportamientos de ciudadanía organizacional están muy relacionados. Existe evidencia de que la percepción de justicia organizacional es la variable que, en mayor medida, predice los comportamientos de ciudadanía organizacional (Colquitt et al., 2001; Hernández \& Yáber, 2009; Karriker \& Williams, 2009; Moorman, 1991; Organ et al., 2005).

Al mismo tiempo, existe evidencia que demuestra que los empleados engaged tienden a dar la milla extra ${ }^{2}$ en su trabajo. Es decir, ponen de manifiesto conductas de desempeño extrarrol (i. e., comportamientos de ciudadanía organizacional) que van más allá de las exigencias del puesto de trabajo. En un estudio realizado por Salanova et al. (2005), encontraron que el engagement de los empleados está relacionado con la percepción que tienen los clientes del desempeño extrarrol de los empleados. De igual forma, este desempeño extrarrol está rela-

2 El término 'dar la milla extra' se refiere a hacer más de lo que es requerido. El concepto de la milla extra ha sido utilizado por múltiples autores para referirse a las comportamientos de ciudadanía organizacional (véanse Organ, 1988,1997; van Dick, Grojean, Christ \& Wieseke, 2006). cionado positivamente con la intención de regreso de los clientes (fidelidad). En otro estudio realizado con enfermeras Salanova et al. (2011), hallaron una relación positiva entre el engagement en el trabajo y los comportamientos extrarrol, los cuales son considerados una variante de los comportamientos de ciudadanía organizacional. Del mismo modo, Babcock-Roberson y Strickland (2010) también registraron una relación positiva entre el engagement y los comportamientos de ciudadanía organizacional.

Moliner et al. (2008) examinaron el rol mediador del bienestar en el trabajo (bajos niveles de burnout y altos niveles de engagement) en las percepciones de justicia organizacional y el desempeño extrarrol en un grupo de empleados del sector de servicios. Este estudio reportó que el engagement mediaba la relación entre justicia organizacional y el desempeño extrarol. Sin embargo, cabe señalar que en esta investigación solo se estudia el rol mediador del engagement (vigor y dedicación) en la relación de la justicia organizacional y los comportamientos extrarrol. Por tanto, solo se toman en cuenta los comportamientos de cortesía y concienciación, las cuales son dimensiones propuestas por Bettencourt y Brown (1997) y Maxham y Netemeyer (2003).

En el presente estudio se pretende examinar el papel que juega el engagement en el trabajo en la relación entre la justicia organizacional y las cinco dimensiones de los comportamientos de ciudadanía organizacional propuestas por Organ (1997) (Altruismo, Concienciación, Deportividad, Cortesía y Virtud Cívica). Se sabe por los hallazgos de Moliner et al. (2008) que el engagement media la relación entre las percepciones de justicia y la conducta extrarrol (cortesía y concienciación), pero todavía queda por explorar si esta relación también existe con las otras dimensiones de los CCO (altruismo, deportividad y virtud cívica). Se entiende que mediante este otro conjunto de conductas se pueden generar espacios de trabajo saludables que fomenten el bienestar y el desempeño óptimo.

A partir de los hallazgos antes citados, se pone de manifiesto que cuando los empleados perciben que trabajan en un lugar donde son tratados de forma justa, estos tienden a dar la milla extra en su trabajo (Cohen-Charash \& Spector, 2001; Her- 
nández \& Yáber, 2009; Karatepe, 2011; Karriker \& Williams, 2009). Sin embargo, otras variables de bienestar, tal como el engagement, puede explicar esta relación. Es decir, cuando los empleados se perciben con mayor bienestar, ponen de manifiesto CCO (en todas sus dimensiones). Para esto, nos basamos en dos teorías: la del intercambio social de Blau (1964) y la teoría de la equidad de Adams (1966), así como el Modelo de Healthy and Resilient Organizations (HERO) (Salanova, Llorens, Cifre \& Martínez, en prensa).

\section{Fundamentación teórica}

La teoría del intercambio social de Blau (1964) señala que las personas establecen y mantienen relaciones por el interés personal de recibir y mantener un beneficio relacional, mediante el intercambio social. Partiendo de esta base, se espera que los componentes relacionales de la justicia organizacional (interpersonal e informacional) influyan en el engagement de los empleados, al percibir este intercambio como un recurso social. Por otro lado, se entiende que la teoría de la equidad de Adams (1966) provee las bases para explicar cómo las percepciones de justicia (distributiva y procedimental) que se sustentan en los recursos instrumentales y económicos (e. g., recompensas y/o bonificaciones), también influyan en el engagement de los empleados (como elementos motivadores externos).

Por último, el modelo HERO (Salanova et al, en prensa) ofrece un marco de referencia para entender el proceso relacional entre las variables del estudio. De acuerdo con Salanova (2008), las organizaciones saludables "tienen formas y prácticas de estructurar y gestionar los procesos de trabajo que hace que sus empleados se sientan bien (...) generan resultados relacionados con la excelencia organizacional y mantienen excelentes relaciones con el entorno organizacional y la comunidad" (p. 187). Este modelo heurístico se compone de tres bloques interrelacionados: Recursos y Prácticas Organizacionales Saludables, Empleados Saludables y Resultados Organizacionales Saludables. En el bloque de prácticas y recursos organizacionales saludables se encuentran aquellos que fomentan los recursos de la tarea (claridad de rol, autonomía, retrocomunicación, justicia distributiva y procedimental) y los recursos interpersonales (desarrollo de carrera, canales de comunicación, justicia interpersonal e informacional) que fomentan la vinculación de los empleados con su trabajo y con las personas con las que trabajan (compañeros, supervisores y clientes). En el bloque de empleados saludables se encuentran los recursos psicológicos positivos y los estados de bienestar que poseen los empleados (creencias de eficacia, afecto positivo, resiliencia, engagement en el trabajo, entre otros). Existe evidencia que apoya la relación entre las variables de bienestar y con resultados organizacionales saludables (véanse, Christian, Garza \& Slaughter, 2011). En el bloque de resultados saludables se encuentran los resultados excelentes de la organización tales como: el desempeño de los empleados, la satisfacción y lealtad de los clientes y la responsabilidad social con el entorno extraorganizacional. Enmarcados dentro de modelo HERO, se puede explicar la relación de las variables del estudio, donde la justicia organizacional (recurso y práctica organizacional saludable) está relacionada con el engagement en el trabajo (empleados saludables), que a su vez está relacionado con los CCO (resultados organizacionales saludables). Esta relación está fundamentada donde la justicia organizacional es un elemento cognitivo que fomenta estados afectivos-cognitivos como engagement, que ponen de manifiesto los CCO como la manifestación conductual en esta cadena de procesos.

Tal como se ha señalado, el propósito de este trabajo es estudiar la relación de la justicia organizacional, el engagement en el trabajo y los comportamientos de ciudadanía organizacional, en una muestra multisectorial de empleados en Puerto Rico. Teniendo en cuenta los argumentos expuestos, así como también hallazgos previos, hipotetizamos que:

Hipótesis: La relación entre la justicia organizacional (distributiva, procedimental, interpersonal e informacional) y los comportamientos de ciudadanía organizacional (altruismo, concienciación, deportividad, cortesía y virtud cívica) está total- 
mente mediada por el engagement en el trabajo (vigor, dedicación y absorción).

\section{Método}

\section{Participantes}

La muestra (por conveniencia) estuvo compuesta por 144 participantes (74\% mujeres) mayores de 21 años, que en el momento del estudio estaban empleados. La edad promedio de los participantes es $32.12(D E=10.47)$. La mayoría de los participantes trabajaban en la empresa privada (54\%), principalmente en el sector de servicios (53\%). La mayoría de los participantes poseían un contrato regular de trabajo (75\%). Finalmente, el $18 \%$ de los participantes realizaba tareas de supervisión.

\section{Procedimiento}

Los datos fueron recopilados durante el 2009 y se siguieron varios pasos para esto. Los participantes del estudio fueron contactados personalmente por el investigador principal para invitarles a participar en el estudio. Después de que los participantes consintieran participar en la investigación al firmar una hoja de consentimiento, donde se detallaba la confidencialidad y voluntariedad de su participación, así como la opción de no participar en el estudio, completaron los cuestionarios en papel fuera del horario laboral y se los devolvieron al investigador principal en un sobre cerrado donde no se identificara el nombre de los participantes. En todo momento, se garantizó la confidencialidad y la voluntariedad de los participantes. Los datos de cada cuestionario y hoja de datos demográficos fueron vaciados en una plantilla de datos y analizados con los programas estadístico SPSS v.21 y PLS Graph (Chin, 2001).

\section{Medidas}

\section{Justicia Organizacional}

Se utilizaron las cuatro dimensiones de la Escala de Justicia Organizacional de Colquitt (2001). Este instrumento consta de 20 ítems que mide las cuatro dimensiones de la justicia organizacional con una escala de respuesta de cinco anclajes que van desde 1 (poco) hasta 5 (mucho). A continuación se presentan algunos ejemplos de ítems para cada subescala: Justicia Distributiva: "iLos resultados alcanzados por Ud. reflejan el esfuerzo que pone en su trabajo?"; Justicia Procedimental: "iUd. tiene influencia sobre los logros obtenidos por la aplicación de las reglas y procedimientos empleados?"; Justicia Interpersonal: "iLa persona que toma decisiones en mi trabajo lo trata con cortesía?"; Justicia Informacional: "iLa persona responsable de llevar a cabo los procedimientos ha sido sincera con Ud.?"

\section{Engagement en el trabajo}

Se midió el engagement utilizando las tres dimensiones de la versión en español del Utrecht Work Engagement Scale de Schaufeli, Bakker y Salanova (2006). Este instrumento consta de nueve ítems (tres ítems para cada dimensión del engagement) que se contestan en una escala de respuesta de siete anclajes va desde 0 (nuncal nada) hasta 6 (siempre/todos los días). Algunos ejemplos de ítems para cada subescala son: Vigor: "En mi trabajo me siento lleno de energía"; Dedicación: "Estoy entusiasmado con mi trabajo"; Absorción: "Estoy inmerso en mi trabajo".

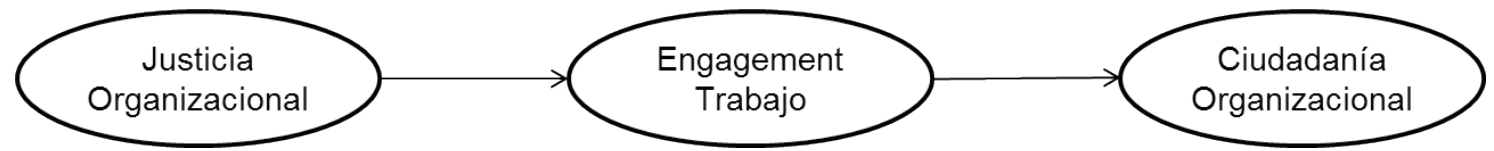

Figura 1. Modelo de hipotetizado de la relación entre justicia organizacional, engagement en el trabajo y los comportamientos de ciudadanía organizacional

Fuente: elaboración propia 


\section{Comportamientos de ciudadanía organizacional}

Se midieron los CCO utilizando las cinco dimensiones propuestas por Organ (1997) (altruismo, concienciación, virtud cívica, deportividad y cortesía) incluidas en la versión corta de Escala Comportamiento de Ciudadanía Organizacional de Martínez Lugo y cols. (2004). Este instrumento consta de 25 ítems, con cinco ítems por cada dimensión, que se contestan en una escala de respuesta tipo Likert de seis anclajes que va desde 0 (totalmente en desacuerdo) hasta 6 (totalmente de acuerdo). Algunos ejemplos de ítems para cada sub-escala son: Altruismo: "Cuando ingresa un empleado nuevo tomo la iniciativa de ayudarle a que se integre a la organización"; Concienciación: "Respeto las normas organizacionales"; Virtud cívica: "Defiendo a mi organización cuando la critican”; Deportividad: "Aun cuando haya interrupciones, termino mi trabajo"; Cortesía: "Informo de antemano cuando voy a ausentarme".

\section{Resultados}

\section{Análisis de datos}

Una vez recopilados los cuestionarios, se introdujeron los datos en una plantilla de SPSS. Todas las medidas del estudio fueron analizadas a partir de las percepciones individuales de cada individuo sobre cada uno de los constructos. Debido a que los cuestionarios tienen diferentes anclajes de respuesta, se utilizaron las puntuaciones estandarizadas de todas las escalas para los análisis estadísticos. Se realizaron análisis descriptivos (medias y desviaciones estándar), correlaciones, análisis de la varianza (ANOVA) con de las escalas con las variables demográficas (género, tipo de organización, tipo de industria y tareas de supervisión), pero no se encontraron diferencias significativas en las medias de las escalas.

Para poner a prueba la hipótesis del estudio, se empleó el modelamiento con ecuaciones estructurales con Partial Least Squares (PLS). Este método de estimación ofrece múltiples ventajas. Una de las ventajas del análisis con PLS es que permite realizar estimaciones entre las variables utilizando un algoritmo interactivo no lineal de mínimo de cuadrados parciales para componentes principales, lo que no hace necesario tener un elevado número de observaciones (Chin \& Newsted, 1999). En estudios de simulación Monte Carlo (Chin, Marcolin \& Newsted, 2003; Chin \& Newsted, 1999) encontraron que las estimaciones realizadas con factores latentes con PLS son estables y fiables con muestras tan pequeñas como con 50 observaciones. A su vez, el método de PLS realiza estimaciones más robustas y conservadoras donde no se utiliza la matriz de varianzas-covarianzas, por lo que no depende muestras muy grandes ni de que los datos estén distribuidos normalmente (Chin, 2001).

Es importante destacar que los modelos de ecuaciones estructurales donde se utilizan la matriz de covarianzas "tratan de minimizar las diferencias entre las muestras de covarianzas y aquellas predichas por el modelo teórico (...) por tanto, el proceso de estimación de parámetros trata de reproducir la matriz de covarianzas con las medidas observadas" (Chin \& Newsted, 1999, p. 309). A diferencia de los modelos basados en las covarianzas, la estimación con PLS se enfoca en maximizar la varianza de las variables dependientes que están explicadas por las variables independientes, en vez de enfocarse en la reproducción empírica de la matriz de covarianzas (Haenlein \& Kaplan, 2004). Es por esta razón que los modelos de ecuaciones estructurales con PLS no poseen índices de ajuste para los modelos, a diferencia de los modelos de ecuaciones estructurales basados en las covarianzas. Otro aspecto importante por destacar es que los modelos de PLS utilizan la técnica de estimación de bootstrapping para determinar si los coeficientes de regresión del modelo estructural son estadísticamente significativos. Esto permite hacer múltiples muestreos de las observaciones y estimar la significancia del efecto en cada coeficiente de regresión entre las variables (Chin \& Newsted, 1999).

Cabe destacar que en nuestro estudio no utilizamos el test de Sobel para probar la mediación, debido a que esta prueba estadística se basa en los supuestos de la estadística paramétrica basada en la matriz de varianza-covarianza. Debido a que en 
utilizamos el método de estimación robusta como el PLS, no es necesario utilizar el test de Sobel para probar si la mediación es estadísticamente significativa. Para ello utilizamos el método de bootstrapping siguiendo las recomendaciones de Chin (2001).

\section{Análisis descriptivos}

La Tabla 1 presenta las medias, desviaciones estándar, alfas de Cronbach e intercorrelaciones de las variables, mientras que en la Tabla 2 se presentan las correlaciones entre los factores latentes, los coeficientes de fiabilidad compuesta y la varianza media extraída de cada factor latente. Tal y como se esperaba, todas las intercorrelaciones del estudio son positivas y significativas. Las correlaciones entre las subescalas de justicia organizacional, engagement en el trabajo y los CCO oscilaron entre 0.16 y 0.82 . Mientras que las correlaciones entre los factores latentes oscilaron entre 0.60 y 0.69 . Para probar si pudiera existir influencia del método común de la varianza, se llevó a cabo la prueba de Harman del factor único mediante un análisis de factores exploratorio de factores, utilizando el método factorización de ejes principales, siguiendo las recomendaciones de Ylitalo (2009). Todos los ítems de los tres cuestionarios fueron incluidos en el análisis y se forzó la extracción de un solo factor. El porciento de la varianza extraída por este único factor fue de $28.93 \%$, lo que está muy por debajo del valor de tolerancia de 58.3\% recomendado por Ylitalo (2009) cuando se tienen menos de 250 observaciones y se realizan análisis con PLS. Por tanto, en nuestra muestra el método común de la varianza no es un problema.

TABLA 1

Medias $(\mathrm{M})$, desviaciones estándar $(\mathrm{DE})$ e intercorrelaciones del estudio $(\mathrm{N}=144)$

\begin{tabular}{|c|c|c|c|c|c|c|c|c|c|c|c|c|c|c|}
\hline & $M$ & $D E$ & 1 & 2 & 3 & 4 & 5 & 6 & 7 & 8 & 9 & 10 & 11 & \\
\hline $\begin{array}{l}\text { Justicia } \\
\text { procedimental }\end{array}$ & 2.59 & 0.8 & $(0.85)$ & & & & & & & & & & & \\
\hline Justicia distributiva & 3.44 & 0.66 & $0.32 * *$ & $(0.75)$ & & & & & & & & & & \\
\hline $\begin{array}{l}\text { Justicia } \\
\text { interpersonal }\end{array}$ & 3.42 & 0.72 & $0.42 * *$ & $0.35^{*}$ & $(0.86)$ & & & & & & & & & \\
\hline $\begin{array}{l}\text { Justicia } \\
\text { informacional }\end{array}$ & 2.94 & 0.96 & $0.53 * *$ & $0.35 * *$ & $0.58 * *$ & $(0.91)$ & & & & & & & & \\
\hline Vigor & 4.44 & 1.22 & $0.49 * *$ & $0.48 * *$ & $0.44 * *$ & $0.42 * *$ & $(0.92)$ & & & & & & & \\
\hline Dedicación & 4.78 & 1.27 & $0.52 * *$ & $0.54 * *$ & $0.5^{* *}$ & $0.60 * *$ & $0.82 * *$ & $(0.93)$ & & & & & & \\
\hline Absorción & 4.55 & 1.38 & $0.43 * *$ & $0.39 * *$ & $0.40 * *$ & $0.53 * *$ & $0.67 * *$ & $0.82 * *$ & $(0.89)$ & & & & & \\
\hline Altruismo & 5.44 & 0.74 & $0.27 * *$ & $0.16^{* *}$ & $0.32 * *$ & $0.48 * *$ & $0.29 * *$ & $0.36 * *$ & $0.38 * *$ & $(0.87)$ & & & & \\
\hline Concienciación & 5.75 & 0.32 & $0.18 * *$ & $0.3 * *$ & $0.2 *$ & $0.22 * *$ & $0.33 * *$ & $0.49 * *$ & $0.46 * *$ & $0.28 * *$ & $(0.76)$ & & & \\
\hline Virtud cívica & 5.22 & 0.87 & $0.44 * *$ & $0.34 * *$ & $0.47 * *$ & $0.47 * *$ & $0.51 * *$ & $0.65 * *$ & $0.62 * *$ & $0.5^{* *}$ & $0.51 * *$ & $(0.88)$ & & \\
\hline Deportividad & 5.24 & 0.81 & $0.36 * *$ & $0.25 * *$ & $0.32 * *$ & $0.49 * *$ & $0.52 * *$ & $0.58 * *$ & $0.55^{* *}$ & $0.71 * *$ & $0.49 * *$ & $0.66^{* *}$ & $(0.8)$ & \\
\hline Cortesía & 5.29 & 0.96 & $0.35 * *$ & $0.32 * *$ & $0.41 * *$ & $0.44 * *$ & $0.42 * *$ & $0.51 * *$ & $0.44 * *$ & $0.30 * *$ & $0.34 * *$ & $0.51 * *$ & $0.5 * *$ & $(0.77)$ \\
\hline
\end{tabular}

Nota. Correlaciones; $p<0.01 * * ; p<0.05^{*}$. Valores alfa para cada una de las escalas están presentados entre paréntesis.

Fuente: elaboración propia

TABLA 2

Correlaciones entre los factores latentes, los Coeficientes de Fiabilidad Compuesta (CFC) y Varianza media extraída (AVE)

\begin{tabular}{llllll}
\hline Variables & CFC & AVE & 1 & 2 & 3 \\
\hline Justicia organizacional & 0.84 & 0.57 & $(0.75)$ & & \\
Engagement en el trabajo & 0.94 & 0.83 & 0.69 & $(0.91)$ & \\
Comportamientos de ciudadanía organizacional & 0.87 & 0.58 & 0.6 & 0.69 & $(0.76)$ \\
\hline
\end{tabular}

Nota. Los elementos diagonales son la raíz cuadrada del AVE entre los constructos y sus indicadores, lo que es indicativo de diferenciación entre los constructos. ** Correlación significativa a un nivel de 0.01 .

Fuente: elaboración propia 


\section{Prueba de hipótesis}

Todas las variables del estudio (justicia organizacional, engagement en el trabajo y los comportamientos de ciudadanía organizacional) fueron incluidas como variables latentes en el modelo de ecuaciones estructurales. Siguiendo las recomendaciones de Colquitt (2001) la justicia organizacional fue analizada como una variable de segundo orden compuesta por sus cuatro dimensiones (procedimental, distributiva, interpersonal e informacional). Siguiendo las recomendaciones de Schaufeli et al. (2006) el engagement representado por un factor latente con sus tres dimensiones (vigor, dedicación y absorción). Por último, siguiendo las recomendaciones de Organ (1988) los CCO fueron analizados como un factor latente por sus cinco dimensiones (altruismo, concienciación, virtud cívica, deportividad y cortesía).

Nuestro modelo plantea que la relación entre la justicia organizacional y los comportamientos de ciudadanía organizacional está mediada por el engagement en el trabajo. Siguiendo las recomendaciones de Baron y Kenny (1986), quienes establecen cuatro procesos para poner a prueba la mediación: primero, se encuentró una relación positiva y significativa entre la justicia organizacional y los $\mathrm{CCO}(\beta$ $=0.61, p<0.05)$. En la segunda parte del proceso,

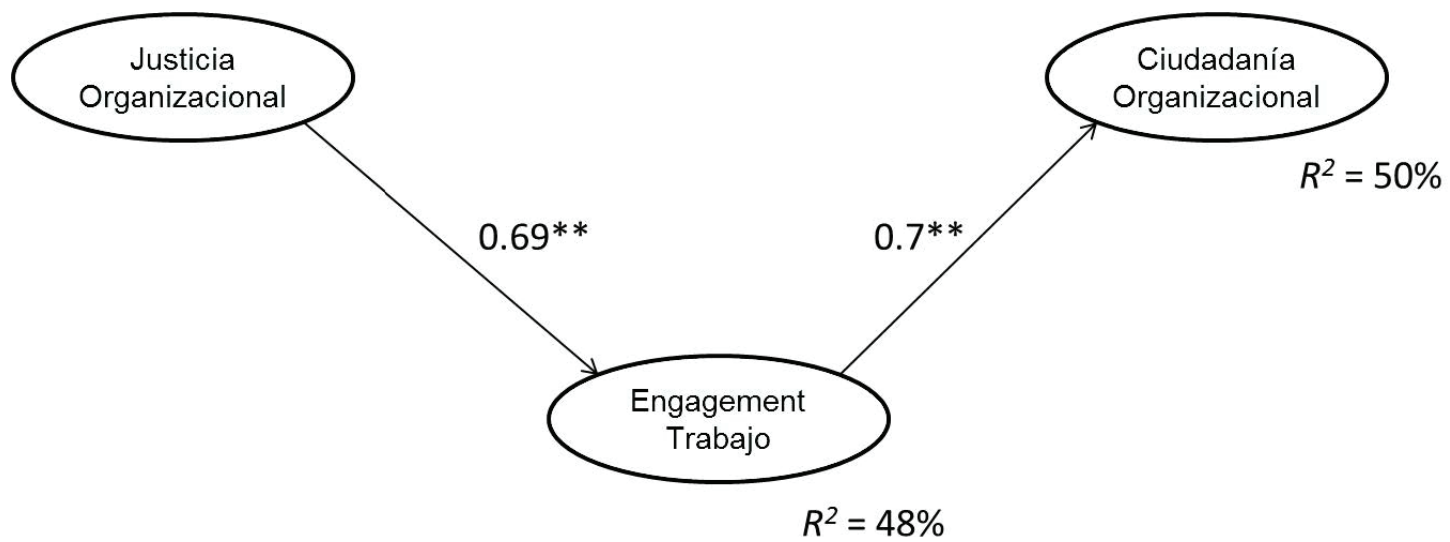

Figura 2. Modelo de mediación total. Los coeficientes presentados en el modelo son estandarizados. Todos los coeficientes son significativos $p<0.001$.

Fuente: elaboración propia

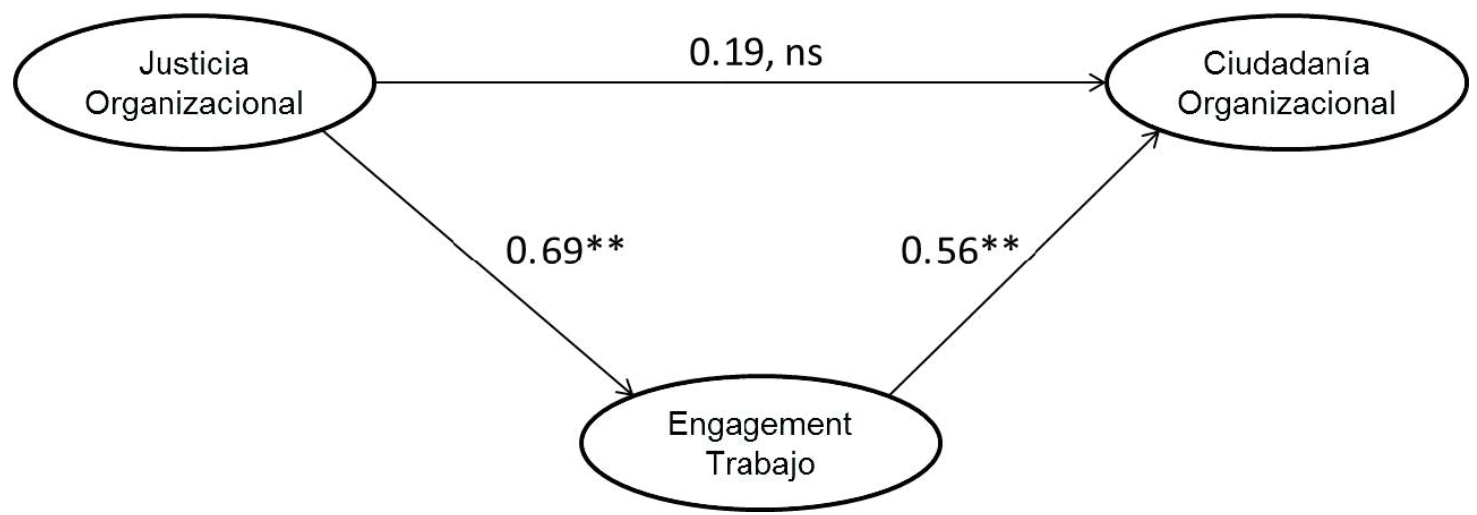

Figura 3. Modelo de mediación parcial. Los coeficientes presentados en el modelo son estandarizados. $n s=p=0.18 . * *=$ $p<0.001$.

Fuente: elaboración propia 
se halló una relación positiva entre la justicia organizacional y el engagement en el trabajo $(\beta=0.7, p$ $<0.05)$. Tercero, se consiguió una relación positiva entre engagement y los $\mathrm{CO}(\beta=0.7, p<0.05)$ y, por último, se puso a prueba el modelo de mediación parcial, y se obtuvo que el coeficiente de regresión entre la justicia organizacional y los $\mathrm{CCO}(\beta=0.19$, $p=0.18)$ dejó de ser estadísticamente significativo al incluir el engagement como mediador. El modelo final evidencia que la justicia organizacional explica un $48 \%$ de la varianza del engagement en el trabajo. Mientras que el engagement en el trabajo explica un $50 \%$ de la varianza de los CCO. Estos resultados evidencian que la relación entre la justicia organizacional y los $\mathrm{CCO}$ en esta muestra está mediada por el engagement en el trabajo. Por tanto, los resultados apoyan la hipótesis propuesta.

\section{Discusión}

El propósito de este estudio es estudiar la relación de la justicia organizacional, el engagement en el trabajo y los comportamientos de ciudadanía organizacional en una muestra multisectorial de empleados, en Puerto Rico. Nuestro modelo de investigación planteaba que el engagement en el trabajo mediaría la relación entre la justicia organizacional y los comportamientos de ciudadanía organizacional, y los resultados apoyan la hipótesis planteada.

\section{Implicaciones teóricas}

Primero, la justicia organizacional está positivamente y significativamente relacionada con el engagement en el trabajo. Es decir, cuando los empleados son tratados de forma justa, equitativa, con dignidad y respeto, estos se podrán sentir más engaged con sus trabajos. Segundo, se pone en evidencia que la justicia organizacional puede ser considerada un recurso organizacional valioso que promueve el engagement en el trabajo. Tercero, también queda demostrado en este estudio que la justicia organizacional y los CCO están positivamente y significativamente relacionados, lo que coincide con hallazgos previos de otras investigaciones (Cohen-Charash \& Spector, 2001; DeConinck, 2010; Karriker \&
Williams, 2009; Moorman, 1991; Niehoff \& Moorman, 1993).

Al mismo tiempo queda evidenciado que el engagement en el trabajo media en su totalidad la relación que existe en la justicia organizacional y los CCO. Creemos que el engagement funciona como mecanismo motivador para que los empleados den la milla extra por la organización. Podemos proveer una posible explicación psicológica para este fenómeno con base en las teorías de equidad de Adams (1966) y el intercambio social del Blau (1964), así como desde el Modelo HERO de Salanova et al. (en prensa).

En primer lugar, cuando los empleados sienten que en su lugar de trabajo: 1) se les trata de forma equitativa y respetuosa; 2 ) se utilizan procesos organizacionales transparentes y justos y 3 ) se les provee información acerca de cómo realizan sus tareas, aumentan las probabilidades de percibir que el intercambio que existe entre el empleado y la organización es justo. Es precisamente a través de esta percepción de intercambio social donde se da la génesis de los CCO. Tal como señalan varios autores (Organ \& Moorman, 1993; Reed \& Kelly, 1993) los CCO emergen de la noción positiva de los empleados sobre intercambio social que se da entre estos y la organización.

En este sentido, Organ y Konovsky (1989) brindan una explicación ontológica a este fenómeno. Según los autores, cuando los individuos se sienten tratados de forma justa por la organización, tienden a reciprocar este sentimiento manifestando CCO hacia la organización. Es importante señalar que para que las personas puedan dar la milla extra por la organización, necesitan experimentar las condiciones necesarias para poder darla. Es precisamente la justicia organizacional la que "asfalta esta carretera psicológica" para que se pongan de manifiesto los CCO. Esto no es algo nuevo en el panorama investigativo, dado que la relación entre la justicia organizacional y los CCO ha sido estudiada en profundidad por múltiples autores (véanse, Colquitt et al., 2001). Sin embargo, los estudiosos de la justicia organizacional han obviado cómo las percepciones de justicia en sus cuatro dimensiones (distributiva, procedimental, interpersonal e informacional) pueden hacer que las personas se sientan más engaged con su trabajo. 
Existe evidencia que demuestra que los recursos laborales incrementan el engagement en el trabajo (Bakker \& Demerouti, 2007; Salanova et al., 2005), del mismo modo este estudio nos permite concluir que las percepciones de justicia organizacional son un vehículo que fomenta e influyen en el bienestar (e. g., el engagement) de los individuos. Nuestro resultados indican que cuando los recursos, tanto de tarea y organizacionales, son provistos de forma equitativa y justa, no solo se promueve el que las personas den más de lo que se les exige en su trabajo, sino que a la vez se fomenta el que las personas se sientan más engaged con su trabajo. Es decir, que den la milla extra sintiéndose bien al hacerlo.

En este sentido Salanova et al. (2005) argumentan que las valoraciones que hacen los empleados a las prácticas que establecen las organizaciones con sus respuestas afectivas y emocionales (e. g., el engagement), sientan la pauta para que las personas den en su trabajo más de lo que se le es requerido en su puesto de trabajo (e. g., los CCO). Ante esto, Halbesleben, Harvey y Bolino (2009) señalan que cuando las personas se sienten a gusto con lo que hacen en su trabajo son más propensas a dar la milla extra, tanto hacia la organización, sus compañeros de trabajo y sus clientes.

Este estudio pone en evidencia que cuando las personas se sienten que son tratadas de forma justa, no solo ponen de manifiesto conductas que favorecen el funcionamiento organizacional, sino que lo hacen sintiéndose más engaged con su trabajo. Es decir, mientras más engaged se siente la persona, mayores $\mathrm{CCO}$ puede manifestar (altruismo, conducta cívica, concienciación, cortesía y deportividad). Además, se evidencia el valor añadido que tienen las percepciones de justicia organizacional, al ser consideradas como un recurso tanto para la organización, así como para los trabajadores.

\section{Implicaciones prácticas}

En cuanto a las implicaciones prácticas de este estudio, queda evidenciado que cuando las personas perciben que las organizaciones para las que trabajan distribuyen los recursos (recompensas e información) de forma equitativa y justa, y se sienten tra- tados con respeto, las personas experimentan mayor bienestar (e.,g., engagement) con su trabajo. Esto a la larga se puede traducir en comportamientos que promuevan el buen funcionamiento de la organización, aun cuando no le son requeridos de forma explícita por su puesto de trabajo (e. g., CCO).

Las organizaciones pueden fomentar los $\mathrm{CCO}$ estableciendo políticas y prácticas organizacionales que sean percibidas como equitativas y justas. Por ejemplo, distribuyendo las recompensas que vayan a la par con los esfuerzos y resultados alcanzados por las personas (justicia distributiva). Estas recompensas pueden ser distribuidas utilizando criterios objetivos para distribuir las recompensas (justicia procedimental). A su vez, para que las recompensas sirvan como un incentivo apropiado, deben ir alineadas a las expectativas que tienen los empleados sobre lo que esperan de su trabajo la organización y sus supervisores. Una práctica común en la gestión del desempeño es la gerencia por objetivos, donde queda plasmado en un plan de trabajo, sobre lo que la organización espera del empleado, así como lo que espera el empleado de la organización.

Entre otras prácticas para incrementar el engagement y los $\mathrm{CCO}$, se pueden incluir el proveer feedback sobre el trabajo que realizan las personas y tomar las decisiones que les afectan, basándose en información objetiva (justicia informacional). Del mismo modo, las políticas organizacionales deben procurar proteger la dignidad y el respeto de las personas (justicia interpersonal). Estas prácticas pueden implantarse a través de la divulgación de información y la formación a los líderes de la organización.

Es importante señalar que el génesis de la justicia organizacional comienza con las prácticas y políticas que establece la organización. Si estas son percibidas como equitativas a partir de las expectativas de los empleados, podrán considerase como un recurso valioso para la organización y sus miembros.

\section{Limitaciones y futuros estudios}

Entre estas se encuentran que esta investigación es de tipo trasversal, por tanto, todas las variables han sido medidas en un solo momento en el tiempo. 
Futuros estudios pueden estudiar las variables de forma longitudinal para poder ver los cambios en las variables a través del tiempo, así como el establecer relaciones de causalidad. Otra posible limitación es el uso de una muestra por disponibilidad, lo que no hace posible generalizar los resultados a otros sectores poblacionales. Sin embargo, es importante destacar que este es el primer estudio de estas variables que se realiza en Puerto Rico. Otra posible limitación puede ser el uso de medidas de autoinforme, por lo que las variables pueden estar influenciadas por el método común de la varianza. Sin embargo, la prueba de Harman para analizar el posible sesgo de la varianza común evidenció que la varianza explicada por el modelo proviene de más de un factor latente, tal como recomienda Ylitalo (2009).

\section{Conclusión}

Estos hallazgos afirman que la justicia organizacional puede ser considerada un buen predictor del engagement en el trabajo, el cual se entiende es un indicador del bienestar de los empleados. Al mismo tiempo, se pone de manifiesto el rol mediador del engagement entre la justicia organizacional y los CCO. Es decir, cuando las personas sienten que son tratadas de forma justa, darán la milla extra por la organización, sus compañeros y sus clientes. Por lo que podemos concluir, es muy probable que cuando una persona es tratada de forma justa en su lugar de trabajo, amará su trabajo y cuando se ama lo que se hace, hacemos más de lo que se nos pide.

\section{Referencias}

Adams, J. S. (1966). Inequity in social exchange. En B. Leonard (Ed.), Advances in experimental social psychology (Vol. 2, pp. 267-299). New York: Academic Press.

Babcock-Roberson, M. E., \& Strickland, O. J. (2010). The relationship between charismatic leadership, work engagement, and organizational citizenship behaviors. Journal of Psychology, 144(3), 313-326.

Bakker, A. B. (2008). Building engagement in the workplace. En C. Cooper \& R. Burke (Eds.), The peak performing (pp. 50-72). Woudestein: Routledge.
Bakker, A. B., \& Demerouti, E. (2007). The Job Demands-Resources model: State of the art. Journal of Managerial Psychology, 22(3), 309-328. doi:10.1108/02683940710733115

Baron, R. M., \& Kenny, D. A. (1986). The moderatormediator variable distinction in social psychological research: Conceptual, strategic, and statistical considerations. Journal of Personality and Social Psychology, 51(6), 1173-1182.

Bettencourt, L. A., \& Brown, S. W. (1997). Contact employees: Relationships among workplace fairness, job satisfaction and prosocial service behaviors. Journal of Retailing, 73(1), 39-61.

Bies, R. J. (1987). Beyond "voice": The influence of decision-maker justification and sincerity on procedural fairness judgments. Representative Research in Social Psychology, 17(1), 3-14.

Blau, P. (1964). Exchange and power in social life. New York: Wiley.

Chin, W. W. (2001). PLS-Graph user's guide. Version 3.0. Houston, TX: Soft Modelling.

Chin, W. W., Marcolin, B. L., \& Newsted, P. R. (2003). A partial least squares latent variable modeling approach for measuring interaction effects: Results from a Monte Carlo simulation study and an electronic-mail emotion/adoption study. Information Systems Research, 14(2), 189-217.

Chin, W. W., \& Newsted, P. R. (1999). Structural equation modeling analysis with small samples using partial least squares. En R. H. Hoyle (Ed.), Statistical strategies for small sample research (pp. 307-341). Thousand Oaks, CA: Sage.

Christian, M. S., Garza, A. S., \& Slaughter, J. E. (2011). Work engagement: A quantitative review and test of its relations with task and contextual. Personnel Psychology, 64(1), 89-136.

Cohen-Charash, Y., \& Spector, P. E. (2001). The role of justice in organizations: A meta-analysis. Organizational Behavior and Human Decision Processes, 86(2), 278-321.

Colquitt, J. (2001). On the dimensionality of organizational justice: A construct validation of a measure. Journal of Applied Psychology, 86(3), 386-400.

Colquitt, J. A., Conlon, D. E., Wesson, M. J., Porter, C. H., \& Ng, K. (2001). Justice at the millenium: A meta-analytic review of 25 years of organizational 
justice research. Journal of Applied Psychology, 86(3), 425-445. http://dx.doi.org/10.1037/00219010.86.3.425

DeConinck, J. B. (2010). The effect of organizational justice, perceived organizational support, and perceived supervisor support on marketing employees' level of trust. Journal of Business Research, 63(12), 1349-1355. http://dx.doi.org/10.1016/j. jbusres.2010.01.003

Farh, J. L., Podsakoff, P. M., \& Organ, D. W. (1990). Accounting for organizational citizenship behavior: Leader fairness and task scope versus satisfaction. Journal of Management, 16(4), 705-721.

Francis, L., Mary, S., \& Barling, J. (2005). Organizational injustice and psychological strain. Canadian Journal of Behavioural Science, 37(4), 250-261.

Greenberg, J. (1987). A taxonomy of organizational justice theories. Academy of Management Review, 12(1), 9-22.

Haenlein, M., \& Kaplan, A. M. (2004). A beginner's guide to Partial Least Squares Analysis. Understanding Statistics, 3(4), 283-297. http://dx.doi. org/10.1207/s15328031us0304_4

Hakanen, J. J., Bakker, A. B., \& Schaufeli, W. B. (2006). Burnout and work engagement among teachers. Journal of School Psychology, 43(6), 495-513. http:// dx.doi.org/10.1016/j.jsp.2005.11.001

Halbesleben, J. R. B., Harvey, J., \& Bolino, M. C. (2009). Too engaged? A conservation of resources view of the relationship between work engagement and work interference with family. Journal of Applied Psychology, 94(6), 1452-1465.

Hernández, I., \& Yáber, G. (2009, junio). Variables socio-demográficas, actitudes laborales y percepción de justicia organizacional como predictores del comportamiento ciudadano universitario. Trabajo presentado en el XXXII Congreso Interamericano de Psicología de la Sociedad Interamericana de Psicología, Guatemala.

Homans, G. (1961). Social behaviour: Its elementary forms. London: Routledge \& Kegan Paul.

Judge, T. A., \& Colquitt, J. A. (2004). Organizational justice and stress: The mediating role of work-family conflict. Journal of Applied Psychology, 89(3), 395-404.

Karatepe, O. M. (2011). Procedural justice, work engagement, and job outcomes: Evidence from Nigeria.
Journal of Hospitality Marketing $\mathcal{E}$ Management, 20(8), 855-878.

Karriker, J. H., \& Williams, M. L. (2009). Organizational justice and organizational citizenship behavior: A mediated multifoci model. Journal of Management, 35(1), 112-135. http://dx.doi. org/10.1177/0149206307309265

Konovsky, M. A., \& Organ, D. W. (1996). Dispositional and contextual determinants of organizational citizenship behavior. Journal of Organizational Behavior, 17(3), 253-266.

Leventhal, G. S. (1976). The distribution of rewards and resources in groups and organizations. En L. Berkowitz \& W. Walster (Eds.), Advances in experimental social psychology (Vol. 9, pp. 91-131). New York: Academic Press.

Loi, R., Yang, J., \& Diefendorff, J. M. (2009). Four-factor justice and daily job satisfaction: A multilevel investigation. The Journal of Applied Psychology, 94(3), 770-781. http://dx.doi.org/10.1037/a0015714

Martínez-Lugo, M., Miranda, S., Donate, J., Benítez, E., Santiago, I., Clavell, A., \& Pagán, A. (2004, noviembre). Desarrollo de la escala de comportamientos de ciudadanía organizacional (ECCO). Cartel presentado en la Quincuagésimo-Primera Convención Anual Asociación de Psicología de Puerto Rico, Ponce, Puerto Rico.

Maxham, J. G., III., \& Netemeyer, R. G. (2003). Firms reap what they show: The effects of shared values and perceived organizational justice on customers' evaluations of complaint handling. Journal of Marketing, 67(1), 46-62.

Moliner, C., Martínez-Tur, V., Ramos, J., Peiró, J. M., \& Cropanzano, R. (2008). Organizational justice and extrarole customer service: The mediating role of well-being at work. European Journal of Work and Organizational Psychology, 17(3), 327-348. http:// dx.doi.org/10.1080/13594320701743616

Moorman, R. H. (1991). Relationship between organizational justice and organizational citizenship behaviors: Do fairness perceptions influence employee citizenship? Journal of Applied Psychology, 76(6), 845-855.

Moorman, R., \& Byrne, Z. S. (2005). How does organizational justice affect organizational citizenship behavior? En J. Greenberg \& J. A. Colquitt (Eds.), 
Handbook of organizational justice (pp. 355-380). New York: Taylor \& Francis.

Moorman, R. H., Niehoff, B. P., \& Organ, D. W. (1993). Treating employees fairly and organizational citizenship behavior: Sorting the effects of job satisfaction, organizational commitment, and procedural justice. Employee Responsibilities and Rights Journal, 6(3), 209-225.

Niehoff, B. P., \& Moorman, R. H. (1993). Justice as a mediator of the relationship between methods of monitoring and organizational citizenship behaviors. Academy of Management Journal, 36(3), 527-556.

Omar, A. (2006). Justicia organizacional, individualismo, colectivismo y estrés laboral. Psicología y Salud, 16(2), 207-217.

Organ, D. W. (1988). Organizational citizenship behavior: The good soldier syndrome. Lexington, MA: Lexington Books.

Organ, D. W. (1997). Organizational citizenship behavior: It's construct clean-up time. Human Performance, 10(2), 85-97.

Organ, D. W., \& Konovsky, M. A. (1989). Cognitive versus affective determinants of organizational citizenship behavior. Journal of Applied Psychology, 74(1), 157-164.

Organ, D. W., \& Moorman, R. H. (1993). Fairness and organizational citizenship behavior: What are the connections? Social Justice Research, 6(1), 5-18.

Organ, D., Podsakoff, P., \& Mackenzie, S. B. (2005). Organizational citizenship behavior: Its nature, antecedents, and consequences. Thousand Oaks: Sage.

Reed, T. F., \& Kelly, D. (1993). An exchange theory of organizational citizenship behavior. En G. R. Ferris (Ed.), Research in personnel and human management (Vol. 11, pp. 41-82). Greenwich, CT: JAI Press.

Ryan, A. (1993). Justice. Oxford, England: Oxford University Press.

Salanova, M. (2008). Organizaciones saludables y desarrollo de recursos humanos. Revista de Trabajo y Seguridad Social, 303(47), 179-214.

Salanova, M., Agut, S., \& Peiró, J. M. (2005). Linking organizational resources and work engagement to employee performance and customer loyalty: The mediation of service climate. Journal of Ap- plied Psychology, 90(6), 1217-1227. http://dx.doi. org/10.1037/0021-9010.90.6.1217

Salanova, M., Llorens, S., \& Schaufeli, W. B. (2011). Yes, I can, I feel good, and I just do it! On gain cycles and spirals of efficacy beliefs, affect, and engagement. Applied Psychology: An International Review, 60(2), 255-285.

Salanova, M., Llorens, S., Cifre, E., \& Martínez, I. M. (en prensa). We need a hero! Towards a validation of the Healthy \& Resilient Organization (HERO) Model. Group E Organization Management.

Salanova, M., Lorente, L., Chambel, M. J., \& Martínez, I. M. (2011). Linking transformational leadership to nurses' extra-role performance: The mediating role of self-efficacy and work engagement. Journal of Advanced Nursing, 67(9), 2256-2266. http:// dx.doi.org/10.1111/j.1365-2648.2011.05652.x

Schaufeli, W. B., Bakker, A. B., \& Salanova, M. (2006). The measurement of work engagement with a short questionnaire. Educational and Psychological Measurement, 66(4), 701-716. http://dx.doi. org/10.1177/0013164405282471

Schaufeli, W. B., Salanova, M., González-Romá, V., \& Bakker, A. B. (2002). The measurement of engagement and burnout: A two sample confirmatory factor analytic approach. Journal of Happiness Studies, 3(1), 71-92.

Thibaut, J., \& Walker, L. (1975). Procedural justice: A psychological analysis. Hillsdale, NJ: Erlbaum.

van Dick, R., Grojean, M. W., Christ, O., \& Wieseke, J. (2006). Identity and the extra mile: Relationships between organizational identification and organizational citizenship behaviour. British Journal of Management, 17(4), 283-301. http://dx.doi. org/10.1111/j.1467-8551.2006.00520.x

Van Dierendonck, D., Schaufeli, W. B., \& Buunk, B. P. (2001). Burnout and inequity among human service professionals: A longitudinal study. Journal of Occupational Health Psychology, 6(1), 43-52. http:// dx.doi.org/10.1037/1076-8998.6.1.43

Ylitalo, J. (2009). Controlling for common method variance with partial least squares path modeling: A Monte Carlo study (Mat-2.4108 Independent Research Projects in Applied Mathematics 64353A). Helsinki: Helsinki University of Technology, Department of Mathematics and Systems Analysis. 